\title{
BMJ Open Neuromonitoring with near-infrared spectroscopy (NIRS) in aneurysmal subarachnoid haemorrhage: a systematic review protocol
}

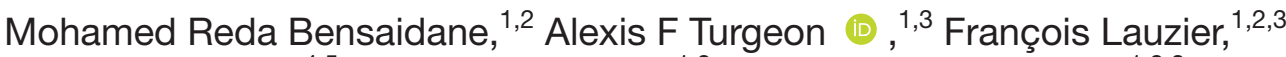 \\ Shane W English, ${ }^{4,5}$ Guillaume Leblanc, ${ }^{1,3}$ Charles L Francoeur (D) 1,2,3
}

To cite: Bensaidane MR, Turgeon AF, Lauzier $\mathrm{F}$, et al. Neuromonitoring with nearinfrared spectroscopy (NIRS) in aneurysmal subarachnoid haemorrhage: a systematic review protocol. BMJ Open 2020;10:e043300. doi:10.1136/ bmjopen-2020-043300

- Prepublication history and additional materials for this paper is available online. To view these files, please visit the journal online (http://dx.doi. org/10.1136/bmjopen-2020043300).

Received 29 July 2020 Revised 22 September 2020 Accepted 06 0ctober 2020

\section{Check for updates}

(C) Author(s) (or their employer(s)) 2020. Re-use permitted under CC BY-NC. No commercial re-use. See rights and permissions. Published by BMJ.

For numbered affiliations see end of article.

\section{Correspondence to} Dr Charles L Francoeur; charles-langis.francoeur.2@ ulaval.ca

\section{ABSTRACT}

Introduction Aneurysmal subarachnoid haemorrhage (aSAH) is a devastating disease associated with high mortality and morbidity. The main threat to patients is delayed cerebral ischaemia (DCl). Near-infrared spectroscopy (NIRS) is a recent technology allowing continuous, non-invasive cerebral oximetry that could permit timely detection of impending $\mathrm{DCl}$ and appropriate intervention to improve outcomes. However, the ability of regional oxygen saturation to detect $\mathrm{DCl}$, its association to the outcome, or benefits of any interventions based on NIRS data, are lacking. Our aims are to evaluate NIRS technology both as a therapeutic tool to improve outcomes in aSAH patients and as a diagnostic tool for DCl. Methods and analysis MEDLINE, EMBASE, Web of Science, the Cochrane Central Register of Controlled Trials and the Cochrane Database of Systematic Reviews will be searched from their inception and without language restriction. Our search strategy will cover the themes of subarachnoid haemorrhage and cerebral oximetry, without limitations regarding studied outcomes. We will identify all observational and interventional human studies of adult patients hospitalised after aSAH that were monitored using NIRS. Functional outcome measures, including the modified Rankin Scale, the Glasgow Outcome Scale and the Barthel Index, will constitute the primary outcome. The Cochrane Risk of Bias tool will be used for randomised controlled trials, the ROBINS-I tool to assess nonrandomised studies of interventions and the NewcastleOttawa Scale for cohort or case-control studies. The Quality Assessment of Diagnostic Accuracy Studies-2 will be applied to studies evaluating NIRS diagnostic accuracy for $\mathrm{DCl}$. We will evaluate the quality of the evidence of the effect based on the Grading of Recommendations Assessment, Development and Evaluation methodology. Ethics and dissemination Dissemination will proceed through submission for journal publication, trial registry completion and abstract presentation. Ethics approval is not required.

PROSPERO registration number CRD42020077522.

\section{INTRODUCTION}

Aneurysmal subarachnoid haemorrhage $(\mathrm{aSAH})$ is a devastating disease accounting for only $5 \%$ of all strokes, ${ }^{1}$ but associated with
Strengths and limitations of this study

- First systematic review on the use of near-infrared spectroscopy in aneurysmal subarachnoid haemorrhage (aSAH) patients.

- Rigorous methodology, in accordance with current guidelines, without language restriction, with a registered and published protocol.

- Two independent reviewers in each phase using specific and well-recognised risk of bias assessment tools.

- Expected low-quality studies.

- Lack of consistency in outcome measures in aSAH patients and evolving definition of delayed cerebral ischaemia in the literature

a mortality rate of up to $45 \%$ and leaving less than half of the survivors independent with activities of daily living. ${ }^{2}$ The main threat to survivors of the initial bleed is a complex syndrome known as delayed cerebral ischaemia (DCI $)^{3}$ occurring in one-third of patients and leading to an increase in case fatality as well as poor functional outcomes. Although the underlying pathobiological processes of DCI are not well understood, they are thought to culminate in brain tissue ischaemia, and hence the long-term poor functional outcome and even death affecting the majority of severe aSAH. It is assumed that early DCI intervention positively affects the outcome. Unfortunately, the lack of adequate neurological examination in highgrade $\mathrm{SAH}$ precludes timely detection of ischaemia and therefore appropriate intervention. To circumvent this situation, most intensive care units (ICU) rely on multiple monitoring modalities for early detection of cerebral changes. These are usually either invasive, unreliable or static. ${ }^{4}$

Near-infrared spectroscopy (NIRS) is a recent technology purportedly allowing 
continuous, real-time, non-invasive cerebral oximetry, thereby allowing detection of brain ischaemia. It provides regional cortical saturation $\left(\mathrm{rSO}_{2}\right)$, a reflection of the balance between oxygen delivery and utilisation in a given region and has been shown to correlate well with jugular venous bulb saturation and brain tissue oxygen pressure. ${ }^{5}$ Clinical studies suggest that it is reliable in detecting perioperative cerebral oxygen desaturation events, especially in cardiac surgery, although clinical benefits remain to be proven. ${ }^{6}$ Although promising in the ICU environment and in the aSAH population, data are still limited. ${ }^{78}$ Threshold $\mathrm{rSO}_{2}$ values associated with poor outcomes or that should trigger intervention are poorly defined and findings associated with one specific device do not necessarily apply to other devices on the market.

Although DCI has been the focus of much work, the ultimate objective is to improve outcomes in aSAH patients. Nimodipine notwithstanding, ${ }^{9}$ trials have so far failed to identify specific therapies fulfilling this goal. ${ }^{4}$ Despite the lack of evidence, many critical care units around the world now routinely use NIRS technology to guide interventions in this population. However, the demonstration of an association of $\mathrm{rSO}_{2}$ parameters to the outcome, or benefits of any interventions based on NIRS data, is lacking. This gap in knowledge needs urgently to be addressed before widespread use of NIRS as a tool to improve outcomes could be recommended.

\section{Objectives}

The present review, therefore, has two distinct objectives:

1. To evaluate NIRS technology as a therapeutic tool to improve outcomes in aSAH patients. To realise this first goal, we will

a. Examine the association between $\mathrm{rSO}_{2}$ values and patient-centred outcomes in the aSAH patient population.

b. Defining the most appropriate threshold that should trigger further investigations or therapeutic intervention, including absolute $\mathrm{rSO}_{2}$ values, changes from the baseline or discrepancy with the contralateral side.

c. Evaluate NIRS-based intervention protocols in this specific clinical setting.

2. To evaluate NIRS technology as a diagnostic tool for DCI. To realise this second goal, we will

a. Determine its validity, reliability and accuracy in detecting DCI.

b. Examine data comparing NIRS to other monitoring modalities for DCI detection.

\section{METHODS AND ANALYSIS}

\section{Design}

The research question and study design were developed by a multidisciplinary team of intensivists, neurologists, health information specialists and epidemiologists. This review will be conducted in accordance with The Cochrane Collaboration principles for Systematic Reviews, ${ }^{10}$ the
Cochrane Methods for Screening and Diagnostic tests ${ }^{11}$ and reported following PRISMA guidelines. ${ }^{12}$

\section{Information sources and search strategy}

MEDLINE, EMBASE, Web of Science, Google Scholar, OpenGrey, the Cochrane Central Register of Controlled Trials, and the Cochrane Database of Systematic Reviews will be searched from their inception.

Conference proceedings from the last 2 years for the following meetings will be reviewed manually: American Association of Neurological Surgeons' Annual scientific meeting, Congress of Neurosurgical Surgeons, Critical Care Canada Forum, the International Symposium on Intensive Care and Emergency Medicine, the International Stroke Conference, Neurocritical Care Society Meeting, the Society of Critical Care Medicine Congress, and World Congress of Neurosurgery. Google Scholar US National Institutes of Health Ongoing Trials Register ( ClinicalTrials.gov), the controlled-trials.com registry, the Trials Central database, Stroke Trials Registry, ISRCTN Registry, Database of Research in Stroke and WHO International Clinical Trials Registry Platform will also be searched for ongoing or unpublished trials. The search will be updated before submission for publication.

Our search strategy will cover the themes of subarachnoid haemorrhage and cerebral oximetry, without limitations regarding studied outcomes. There will be no language restriction. It will use Medical Subject Headings $(\mathrm{MeSH})$ terms to capture each of the principal elements of the research question, along with appropriate keywords. The proposed MEDLINE search strategy is available (see online supplemental appendix 1) and will be adapted for the other databases.

\section{Study selection}

\section{Population}

We will include all original observational and interventional human studies, including randomised controlled trials (RCT), non-randomised studies of intervention and prospective and retrospective observational studies, of adult patients ( $\geq 18$ years old) hospitalised after aSAH. In the case of studies on mixed populations, we will exclude studies were aSAH patients constitute less than $80 \%$ of the studied population and will apply the same approach for the age criteria.

\section{Intervention and Control}

We will include studies using NIRS monitoring during the index hospitalisation, either as a diagnostic, prognostic or therapeutic tool or in a blinded fashion. We will exclude studies where NIRS monitoring is limited to the time of aneurysm surgery. A control group is not required for inclusion.

\section{Outcome}

Inclusion in our systematic review will not be limited based on reported outcome, although our analyses will focus on functional outcome and measures of test utility as described in the methodology section. 
We will use Covidence ${ }^{13}$ as a citation manager and remove all duplicates. Two reviewers (MRB and CF) will independently screen retrieved citations by title and abstract to exclude irrelevant studies. Remaining citations will undergo a full article review to assess eligibility for inclusion by the same independent reviewers. Discordance will be resolved by consensus and when necessary in consultation with a third independent senior reviewer (SE).

\section{Data collection}

The same reviewers will independently extract data from eligible studies using a standardised, pilot-tested data extraction form developed using Research Electronic Data Capture (REDCap), ${ }^{14}$ a web-based tool. Discordance will be resolved by consensus and if necessary in consultation with a third reviewer (SE). The information collected will include:

1. Study characteristics: title, authors, journal/source, year and language of publication, country, type of study, total number of patients, inclusion/exclusion criteria, DCI definition and ascertainment, randomisation, allocation concealment, blinding methods (where applicable) and financial support.

2. Population characteristics: age, gender distribution, clinical setting (ICU or other) aSAH clinical grade (World Federation of Neurological Surgeons grading scale, Hunt and Hess grading system, Glasgow Coma Scale) and imaging severity (Fisher scale, Claassen CT rating scale), aneurysm size and location, elevated intracranial pressure.

3. Exposure and/or interventions of interest: number of patients monitored with NIRS or in an intervention arm, specific cerebral oximetry device used, location of NIRS sensors, timing and duration of monitoring, data quality (missing monitoring data, monitoring interruptions, inability to monitor), $\mathrm{rSO}_{2}$ threshold for intervention or investigation, $\mathrm{rSO}_{2}$ metrics (means, medians, dispersion), $\mathrm{rSO}_{2}$ threshold (s) with best predictive accuracy according to authors as well as a detailed description of all elements of NIRS-based intervention protocols if any (ie, any investigation or any treatment triggered or based on NIRS parameters).

4. Cointerventions of interest: haemodynamic support, invasive ventilatory support, nimodipine use, intracranial pressure monitoring and intracranial hypertension treatment, red blood cells transfusions, surgical or endovascular aneurysm treatment, endovascular treatment of vasospasm, medical DCI management.

5. Outcomes: there is a lack of consistency in outcome measures reported in aSAH, and a core outcome set has yet to be developed. ${ }^{15}{ }^{16}$ We will focus on functional outcome measures as our primary outcome, evaluated with the modified Rankin Scale (mRS), the Glasgow Outcome Scale (GOS) and its extended version (GOSe) or the Barthel Index, at 90 days or more. Secondary outcomes will also be collected and include any functional outcome at fewer than 90 days, cogni- tive outcomes as measured with neuropsychological tests such as the Mini-Mental State Examination, the Montreal Cognitive Assessment or other validated metrics, quality-of-life outcomes as measured by patientreported outcome instruments such as the Short Form (SF-12 or SF-36) Health Survey, the EuroQol fivedimensional (EQ-5D) score or other validated metric, early and late mortality (before and after 90 days), incidence of DCI, incidence of vasospasm (detected by transcranial Doppler, CT or digital subtraction angiography), length of stay in hospital and of mechanical ventilation. DCI definitions and adjudication mechanisms will also be extracted.

For observational studies, both crude and adjusted estimates of outcomes will be extracted. The confounders adjusted for will be reported.

6. Diagnostic accuracy and comparators for DCI detection: description of reference standard for diagnosis of DCI, true and false positives and negatives, sensitivity, specificity, positive predictive value and negative predictive value for DCI as reported by authors, receiver operating characteristics (ROC) curve, likelihood ratios for the respective test results, description of comparators with applied criteria if any, including transcranial Dopplers, CT or digital subtraction angiography, invasive brain oxygen monitoring, microdialysis, invasive or non-invasive cerebral blood flow monitoring, quantitative or raw Electroencephalography (EEG).

Study authors will be contacted for clarification or additional results as needed.

\section{Assessment of methodological quality and risk of bias assessment}

The risk of bias assessment will be performed independently by the same reviewers (MRB and CF), with disagreement resolved by consensus or a third reviewer if necessary. The Cochrane Risk of Bias tool ${ }^{17}$ will be used for RCTs, the Risk Of Bias in Non-randomized Studies - of Interventions (ROBINS-I tool) ${ }^{18}$ to assess non-randomised studies of interventions and the Newcastle-Ottawa Scale for cohort or case-control studies. ${ }^{19}$ The Quality Assessment of Diagnostic Accuracy Studies- $2^{20}$ will be applied to studies evaluating NIRS diagnostic accuracy for DCI.

\section{Data synthesis and analytical plan}

A description of all included studies will first be reported with the aid of tables and text. Both a narrative synthesis and, where possible, a quantitative meta-analysis of the data will be presented. Studies will be clustered according to design (intervention vs observational, randomised vs non-randomised) and main objective (clinical outcome vs DCI detection) and analysed separately. Suitability for meta-analysis will be determined by the degree of heterogeneity (clinical and statistical) observed between the studies. The $\mathrm{I}^{2}$ index will be used to evaluate the presence of statistical heterogeneity. For observational studies, analysis will be conducted with adjusted estimates, and confounding factors taken into account will be detailed. 
We will perform pooled analyses using random effects models with the DerSimonian and Laird method when appropriate. Funnel plot analysis will be used to detect potential reporting bias and the trim-and-fill method ${ }^{21}$ will be applied to allow estimation of the number of missing studies that might exist and adjustment of the overall effect estimate. For diagnostic accuracy studies, summary estimates of sensitivity and specificity and hierarchical ROC curve summary will be presented.

Primary outcome will be converted to a standardised dichotomous variable with an mRS of $0-3$, a GOS of 4-5, a GOSe of $4-8$ and a Barthel Index of $75^{22}$ or more representing a favourable outcome. Results will be presented in tabular format. Secondary outcomes will be analysed according to the data type. Pooled dichotomous outcomes such as mortality will be expressed as risk ratios and presented in tabular format, whereas pooled continuous effect measures will be expressed as mean differences, both with $95 \%$ CIs. Heterogeneity of cognitive and quality-of-life outcome measures will not allow pooling or conversion to a standardised dichotomous outcome across different metrics.

We will use the author's definitions of DCI, and its incidence (excluding case-control and cross-sectional studies) will be defined as the number of patients suffering from DCI during hospitalisation for aSAH divided by the total number of patients hospitalised for SAH (at-risk population). Sensitivity analyses will be conducted by excluding studies where DCI definition relies exclusively on radiological vasospasm and also by including only studies where DCI diagnosis is made based on new infarcts on CT or MRI. We will perform subgroup analyses based on the severity of the injury (high clinical grade on admission constituting the majority of patients or not) and the risk of bias (low vs other risks).

\section{Missing data}

Studies will not be excluded based on missing data. Original investigators will be contacted for any missing information regarding outcomes, summary data, individual or study-level characteristics. Analysis will be performed on available data when data can be assumed to be missing at random. Other missing values will be imputed with replacement values as appropriate and the underlying assumptions reported. Sensitivity analyses will be performed to assess the latter. The potential impact of missing data will be discussed.

\section{Quality of evidence}

We will evaluate the quality of the evidence of the effect based on the Grading of Recommendations Assessment, Development and Evaluation methodology. ${ }^{23}$

\section{DISCUSSION}

Outcomes in SAH patients might intrinsically be associated to early detection of DCI and its proper management. High-grade patients are both at higher risk of DCI and without available clinical examination to detect it. Invasive monitoring is limited by its very local evaluation and most imaging modalities are static, implying deleterious lags in event detection. However, critical care in general, and neurocritical care more specifically, is submerged with monitoring alternatives, most of which have yet to be submitted to meticulous evaluation before incorporating the information they provide to usual patient management. More important, sound evaluation dictates that technology impact on patient-centred outcomes be prioritised. Our systematic review will identify, analyse and summarise the evidence supporting the use of cerebral oximetry monitoring in aSAH patients. It will inform subsequent steps in the design and implementation of further research on the matter, with the ultimate objective of studying the role of cerebral oximetry both as a diagnostic tool and a potential aide to guide DCI therapy and subsequently improve outcomes in this population in dire need of evolution in their management. Potential limitations to our study include expected low-quality studies, a lack of consistency in outcome measures in our population, an evolving definition of DCI in the literature and the absence of a gold standard for DCI diagnosis in high-grade aSAH patients.

\section{ETHICS AND DISSEMINATION}

The study results will be released to the general medical community through submission for publication in a peer-reviewed journal within 3 months of completion, regardless of the magnitude or direction of the reported findings. Trial registry will also be updated with the study results. Finally, the results will be submitted for presentation as an abstract at a national or international conference targeting an appropriate audience (neurology, neurosurgery and/or critical care). Ethics approval is not required.

\section{PATIENTS AND PUBLIC INVOLVEMENT}

The present study focuses on patient-centred outcomes, including survival, functional outcomes and quality of life. No patient was involved in the design of the study protocol.

\section{Author affiliations}

${ }^{1} \mathrm{CHU}$ de Québec - Université Laval Research Center, Population Health and Optimal Health Practices Research Unit (Trauma-Emergency-Critical Care Medicine),

Université Laval, Quebec City, Quebec, Canada

${ }^{2}$ Department of Medicine, Université Laval, Quebec City, Quebec, Canada

${ }^{3}$ Department of Anesthesiology and Critical Care Medicine, Division of Critical Care

Medicine, Université Laval, Quebec City, Quebec, Canada

${ }^{4}$ Clinical Epidemiology Program, Ottawa Hospital Research Institute, Ottawa,

Ontario, Canada

${ }^{5}$ Division of Critical Care, Department of Medicine, University of Ottawa, Ottawa, Ontario, Canada

Funding This work is supported by a Fondation Scheme Grant (\#148449) from the Canadian Institutes of Health Research (CIHR). Dr Turgeon is the chairholder of the Canada Research Chair in Critical Care Neurology and Trauma. Dr Lauzier is a 
recipient of a salary support Award from the Fonds de la Recherche du Québec Santé (FRQS).

Competing interests None declared.

Patient consent for publication Not required.

Provenance and peer review Not commissioned; externally peer reviewed.

Supplemental material This content has been supplied by the author(s). It has not been vetted by BMJ Publishing Group Limited (BMJ) and may not have been peer-reviewed. Any opinions or recommendations discussed are solely those of the author(s) and are not endorsed by BMJ. BMJ disclaims all liability and responsibility arising from any reliance placed on the content. Where the content includes any translated material, BMJ does not warrant the accuracy and reliability of the translations (including but not limited to local regulations, clinical guidelines, terminology, drug names and drug dosages), and is not responsible for any error and/or omissions arising from translation and adaptation or otherwise.

Open access This is an open access article distributed in accordance with the Creative Commons Attribution Non Commercial (CC BY-NC 4.0) license, which permits others to distribute, remix, adapt, build upon this work non-commercially, and license their derivative works on different terms, provided the original work is properly cited, appropriate credit is given, any changes made indicated, and the use is non-commercial. See: http://creativecommons.org/licenses/by-nc/4.0/.

\section{ORCID iDs}

Alexis F Turgeon http://orcid.org/0000-0001-5675-8791

Charles L Francoeur http://orcid.org/0000-0002-8147-8852

\section{REFERENCES}

1 Benjamin EJ, Muntner P, Alonso A, et al. Heart disease and stroke statistics-2019 update: a report from the American heart association. Circulation 2019;139:e56-66.

2 Rinkel GJE, Algra A. Long-term outcomes of patients with aneurysmal subarachnoid haemorrhage. Lancet Neurol 2011;10:349-56.

3 Rosengart AJ, Schultheiss KE, Tolentino J, et al. Prognostic factors for outcome in patients with aneurysmal subarachnoid hemorrhage. Stroke 2007;38:2315-21.

4 Francoeur CL, Mayer SA. Management of delayed cerebral ischemia after subarachnoid hemorrhage. Critical Care 2016;20:1-12.

5 Smith M. Shedding light on the adult brain: a review of the clinical applications of near-infrared spectroscopy. Phil Trans $R$ Soc $A$ 2011;369:4452-69.

6 Serraino GF, Murphy GJ. Effects of cerebral near-infrared spectroscopy on the outcome of patients undergoing cardiac surgery: a systematic review of randomised trials. BMJ Open 2017;7:e016613
7 Arshi B, Mack WJ, Emanuel B. Invasive and noninvasive multimodal bedside monitoring in subarachnoid hemorrhage: a review of techniques and available dats. Neurol Res Int [online] 2013;2013:1-4.

8 Burzyńska M, Uryga A, Kasprowicz M, et al. The relationship between the time of cerebral desaturation episodes and outcome in aneurysmal subarachnoid haemorrhage: a preliminary study. J Clin Monit Comput [online] 2019.

9 Pickard JD, Murray GD, Illingworth R, et al. Effect of oral nimodipine on cerebral infarction and outcome after subarachnoid haemorrhage: British aneurysm nimodipine trial. BMJ 1989;298:636-42.

10 Higgins J, Green S. Cochrane handbook for systematic reviews of interventions. [Internet]. Version 6. The Cochrane Collaboration, 2019. www.cochrane-handbook.org

11 Deeks JJ, Bossuyt PM GC, eds. Cochrane handbook for systematic reviews of diagnostic test accuracy version 1.0.0. The Cochrane Collaboration, 2009

12 Moher D, Shamseer L, Clarke M, et al. Preferred reporting items for systematic review and meta-analysis protocols (PRISMA-P) 2015 statement. Syst Rev 2015;4:1-9.

13 Covidence systematic review software. Veritas health innovation, Melbourne, Australia. Available: www.covidence.org

14 Harris PA, Taylor R, Thielke R, et al. Research electronic data capture (REDCap)-A metadata-driven methodology and workflow process for providing translational research informatics support. J Biomed Inform 2009;42:377-81.

15 Andersen CR, Fitzgerald E, Delaney A. Finfer S. a systematic review of outcome measures employed in aneurysmal subarachnoid hemorrhage (aSAH) clinical research. Neurocrit Care [online] 2018:1-8.

16 Andersen CR, Presseau J, Saigle V, et al. Core outcomes for subarachnoid haemorrhage. Lancet Neurol 2019;18:1075-6.

17 Higgins JPT, Altman DG, Gøtzsche PC, et al. The Cochrane collaboration's tool for assessing risk of bias in randomised trials. BMJ 2011;343:d5928.

18 Sterne JA, Hernán MA, Reeves BC, et al. ROBINS-I: a tool for assessing risk of bias in non-randomised studies of interventions. BMJ 2016;355:i4919.

19 Wells G, Shea B, O'Connell D, et al. The Newcastle-Ottawa scale (NOS) for assessing the quality of nonrandomised studies in metaanalyses, 2013. Available: http://www.ohri.ca/programs/clinical_ epidemiology/oxford.asp

20 Whiting PFet al. QUADAS-2: a revised tool for the quality assessment of diagnostic accuracy studies. Ann Intern Med 2011;155:529-36.

21 Duval S, Tweedie R. Trim and fill: a simple funnel-plot-based method of testing and adjusting for publication bias in meta-analysis. Biometrics 2000;56:455-63.

22 Uyttenboogaart M, Stewart RE, Vroomen PCAJ, et al. Optimizing cutoff scores for the Barthel index and the modified Rankin scale for defining outcome in acute stroke trials. Stroke 2005;36:1984-7.

23 Guyatt GH, Oxman AD, Vist GE, et al. Grade: an emerging consensus on rating quality of evidence and strength of recommendations. $B M J$ 2008;336:924-6. 